\title{
然
}

Marta Alzás de la Fuente*

Margarita Clemente Muñoz**

Paula de la Cruz Rodríguez*

Mercedes Núñez Román ${ }^{* * *}$

\section{LA DIRECCIÓN GENERAL DE POLÍTICA COMERCIAL COMO AUTORIDAD ADMINISTRATIVA DE CITES EN ESPAÑA}

\section{Un modelo de excelencia en colaboración nacional e internacional}

En el año 1973 tuvo lugar la firma del Convenio sobre el Comercio Internacional de Especies Amenazadas de Fauna y Flora Silvestres (CITES) para adoptar una serie de medidas que permitiesen equilibrar el uso sostenible de las especies y la conservación de las mismas. Tras casi medio siglo de vigencia, CITES ha conseguido convertirse en un exitoso instrumento comercial al servicio de la conservación y el uso sostenible de las especies, capaz de combinar de manera eficaz la relación comercio y medio ambiente. En la actualidad son 184 países los que han ratificado el convenio, habiendo sido España el 89..$^{\circ}$ en adherirse a él en el año 1986. Desde entonces y hasta la fecha del 2 de enero de 2022 la Secretaría de Estado de Comercio ha estado al frente de la aplicación de este convenio como autoridad administrativa principal del mismo realizando una encomiable labor ampliamente reconocida a nivel internacional.

Palabras clave: Autoridad Administrativa de España, comercio, SOIVRE.

Clasificación JEL: F18, F13, Q56, Q57, Q58.

\section{Introducción}

La puesta en marcha del Convenio CITES en España fue un cometido de enorme complejidad debido a la poca disponibilidad de

* Jefa de Área CITES.

** Asesora Científica de Flora CITES.

*** Coordinadora Nacional CITES.

Versión de enero de 2022.

DOI: https://doi.org/10.32796/bice.2022.3142.7336 personal y la falta de experiencia en aquel momento. A pesar de ello, se logró poner en funcionamiento gracias al enorme esfuerzo, dedicación y profesionalidad, y a la profunda convicción de los méritos del Convenio CITES como instrumento de política comercial al servicio de la conservación.

Para hacer frente a dicha complejidad dentro y fuera de España y contribuir a la correcta aplicación del convenio, la Secretaría de $D$ 
Marta Alzás, Margarita Clemente, Paula de la Cruz y Mercedes Núñez

Estado de Comercio, en sus funciones como autoridad administrativa CITES, emprendió múltiples actuaciones en distintos ámbitos (formación, cooperación, divulgación, etc.). Algunas de las más relevantes se recogen hoy en el presente documento como un reconocimiento al trabajo de los funcionarios que las abordaron.

\section{Actividades de carácter formativo}

De entre todas, cabe destacar, sin lugar a dudas, las acciones formativas. Estas son las que han tenido mayor repercusión ya que han supuesto un impacto directo en la mejora de la aplicación del convenio, tanto interna como externamente. Estas acciones se han dirigido a las autoridades y órganos implicados en la aplicación y ejecución de CITES con el objetivo principal de capacitarlos para el desarrollo efectivo y eficaz de estas competencias.

La primera de ellas data de 1982, años antes de la implementación legislativa del convenio en España, y materializada mediante la publicación de un pequeño libreto, por parte de la entonces Dirección General de Exportación, en el que se desarrollaba su funcionamiento, las especies que estaban incluidas, las excepciones, los tipos y formatos de reuniones, y las implicaciones del comercio de las especies recogidas, constituyendo así la primera herramienta didáctica y divulgativa de las tantas que, a iniciativa propia, se generarían en los años siguientes.

Ya desde el propio comienzo de la aplicación del Convenio CITES en España, el personal encargado de la coordinación en los servicios centrales del ministerio planificaba, elaboraba y proporcionaba formación a todos los agentes encargados de la aplicación en
España, desde formación general a la Guardia Civil y la formación especializada a los equipos del SEPRONA hasta la formación particular a agentes de aduana y otros sectores interesados, e incluso formación dirigida y adaptada a criadores, comunidades autónomas y policías locales, pues se adoptó el principio de que una correcta implementación del Convenio CITES debía pasar irremediablemente por el completo y preciso conocimiento de todo su contenido, excepciones y posibilidades, por parte de todos los agentes implicados.

\subsection{Material didáctico}

Los funcionarios que formaron y forman parte de la autoridad administrativa CITES española no solo se han especializado y han profundizado en cómo implementar el convenio con sus mejores capacidades, sino que, además, generaron valiosísimas herramientas de identificación, aplicación e interpretación, bajo la tutela del Ministerio y de la Dirección General en la que se encontraban imbricados, pero basados en sus conocimientos y capacidades, y con el único propósito de dar difusión a la experiencia, ayudar a la comprensión de un convenio de estas características y colaborar con el conocimiento común y adaptado.

Ejemplo de ello son publicaciones de la talla de la Guía de identificación de mariposas protegidas por el Convenio de Washington y por la Unión Europea, elaborado por Vicente Roncero Carrochano y Enrique Tormo Muñoz, inspector del SOIVRE y presidente del Grupo Entomológico de Alicante, respectivamente, en 1998, que serviría como guía para la identificación de géneros de mariposas que aún hoy constituye una de las materias más complejas del convenio. 
Del mismo autor que la publicación anterior, Vicente Roncero Carrochano, actual director provincial de Comercio de Alicante, encontramos también la Guía de identificación de conchas y corales incluidos en el Convenio de Washington (CITES), publicada en 2001; sin duda, una de las obras bandera de las publicaciones de la autoridad administrativa española, no solo por lo completa y bien estructurada que se encuentra, sino por su sencillez y perfecta orientación que guía al lector hacia una certera identificación, clasificación y requisitos necesarios para poder encontrar ejemplares de estas especies en el comercio, según los preceptos del Convenio CITES, elementos todos ellos que han facilitado que se imprimieran dos ediciones de esta publicación.

No es posible continuar este apartado sin hacer referencia a la Guía de efectos personales de especies protegidas CITES, elaborado y publicado por José Ruiz Rosa, personal SOIVRE de la Dirección Territorial de Comercio de Barcelona, en el año 2015.

Esta guía, consultada a diario por personal de la autoridad administrativa, agentes de aduanas, personal de las aduanas, viajeros y autoridades de aplicación, tanto en su versión física como en la web, no solo combina a la perfección la información detallada, con imágenes reales recopiladas en las inspecciones de la autoridad administrativa, con el conocimiento de los países de origen y destino más comunes, sino que señala de forma muy precisa aquellos objetos y manufacturas que más se encuentran en comercio fruto de las transacciones de los denominados «efectos personales», brindando al lector información precisa sobre lo que son, cuáles son los más comunes y de dónde proceden.

A pesar de que el hilo cronológico no se haya respetado en este caso, merece una mención de honor, por su complejidad, detalle y elaboración, el Manual de identificación de partes de especies incluidas en Anexos del CITES, elaborado por Blas Vicente López, Carlos Paños Collado (inspectores del SOIVRE de la Dirección Territorial de Comercio de Alicante) y Eugenio Bañón Rodes (fotógrafo), en 1989.

Este manual constituye una verdadera obra de arte en la historia de la autoridad administrativa CITES en España, no solo porque realizó una minuciosa descripción de las especies de serpientes, lagartos, cocodrilos y similares que eran más comercializadas en España, ayudando así a su identificación, sino porque incluyó un capítulo para las imitaciones, y completó la descripción literaria con fotografías reales realizadas durante las inspecciones llevadas a cabo en los primeros años de andadura de la autoridad administrativa.

Pero, sin duda, todo este interés por la formación, la divulgación y la correcta interpretación que ayudara no solo a autoridades, sino también a los propios operadores y empresas del sector, a aplicar correctamente el Convenio CITES, y a cumplir en última instancia con sus objetivos mediante la consecución de un comercio de vida silvestre regulado, sostenible y equilibrado, tendría como culmen el lanzamiento de todo un sueño para muchas autoridades administrativas, e incluso para la propia Secretaría CITES: un máster internacional que aunara todos los conocimientos, la experiencia adquirida y la capacitación para formar a otras autoridades relacionadas con el Convenio CITES.

\subsection{Fichas de reptiles para los manuales de identificación CITES de la Secretaría}

En la VIII Conferencia de las Partes celebrada en Ottawa en 1987, España propuso la $\triangleright$ 
inclusión de tres especies de lacértidos en los apéndices de CITES y con ello adquirió el compromiso de acompañar su propuesta con las fichas de identificación de las correspondientes especies.

Para cumplir con dicho compromiso, la Dirección General de Conservación de la Naturaleza acordó con el profesor Dr. Valentín Pérez-Mellado, de la Universidad de Salamanca, la preparación de la información textual y gráfica necesaria para la edición de las fichas correspondientes a las tres especies.

Una vez completadas se enviaron a la Secretaría CITES por parte de la autoridad administrativa española para ser incluidas en el Manual de Identificación CITES. Este manual conforma una colección de fichas de las distintas especies de fauna y flora incluidas en el convenio y están diseñadas para ayudar en la identificación con ilustraciones detalladas en blanco y negro, mapas de distribución de las poblaciones y descripciones concisas muy valiosas para las partes implicadas en la aplicación y observancia del convenio.

\subsection{El programa Karolus}

Para finalizar este apartado, se hará alusión a una interesante actividad formativa y de intercambio laboral que surgió como iniciativa de la Comisión Europea.

Casi treinta años atrás hubo algunos funcionarios españoles que se aventuraron a poner en práctica sus conocimientos sobre CITES aprovechando la oportunidad de trabajar dentro de la autoridad administrativa de otro Estado miembro. Si bien a día de hoy no existe esta posibilidad, en el año 1999 sí, gracias a la existencia de un programa de formación e intercambio de funcionarios de la Unión Europea, conocido como Karolus.

El programa Karolus se creó en 1992 y uno de sus cometidos fundamentales era fomentar el desarrollo de una política convergente de la aplicación del derecho comunitario en el ámbito del mercado interior, siendo CITES uno de los sectores legislativos señalados como prioritarios. Para lograr este objetivo, el programa estaba estructurado en tres partes, la primera de ellas consistente en la realización de un seminario de formación en Maastricht; la segunda, en la realización de un intercambio en el cual el funcionario seleccionado trabajaría con sus homólogos de otro Estado miembro durante un periodo medio de dos meses; y la tercera, en la asistencia a un seminario de evaluación final.

Otro de los propósitos principales del programa Karolus era crear una red de contactos personales entre los funcionarios de los distintos Estados miembros que ejerciesen en la misma materia. En este sentido, cabe indicar que tanto este como el anterior objetivo fueron plenamente alcanzados por José Ruiz Rosa, quien, con apenas un par de años de experiencia en CITES, trabajó durante un mes en el Puesto de Inspección Fronteriza de Roissy (aeropuerto de París) como parte de la autoridad administrativa CITES de Francia.

Su participación en este intercambio no solo le permitió proporcionar una visión crítica del modelo de funcionamiento francés, cuyas fortalezas y debilidades plasmó en detalle en un informe de especial interés para la S. G. de Inspección, Certificación y Asistencia Técnica del Comercio Exterior, sino que, además, también consiguió mantener por largos años el contacto con algunos de los funcionarios nacionales e internacionales con los que tuvo la oportunidad de coincidir en ese intercambio. 


\section{Actividades de carácter asistencial y cooperativo}

En lo que concierne a la colaboración en el ámbito de las actividades de información, cooperación y de asistencia técnica, la autoridad administrativa española ha mantenido siempre un perfil caracterizado por el apoyo y la asistencia a las empresas del sector. Estas empresas han recibido charlas informativas organizadas ad hoc para los distintos sectores interesados, además de la atención constante y resolución de dudas acerca del CITES.

El máximo exponente de cooperación nacional lo encontramos tras la publicación del Real Decreto 1333/2006, de 21 de noviembre, por el que se regula el destino de los especímenes decomisados de las especies amenazadas de fauna y flora silvestres protegidas mediante el control de su comercio, en el que se fija normativamente, entre otras cuestiones, la importancia de la cooperación con el sector privado, y la designación de centros de rescate de especímenes decomisados, ante la falta de estructuras en este sentido por parte de la propia Administración.

\subsection{Centros de rescate de especímenes decomisados}

A través de las direcciones territoriales y provinciales en cada demarcación, y fruto de los trabajos colaborativos que se habían dado en los últimos años desde la publicación del mencionado real decreto, comienza en 2015 una labor de búsqueda y diálogo con entidades del sector público y privado, como zoológicos, centros de recuperación de especies, centros de investigación y fundaciones para la conservación de especies que pudieran ayudar en la implementación del convenio, en una de sus facetas más críticas y sensibles como era el mantenimiento y alojamiento de los ejemplares vivos que se decomisaran en las operaciones policiales, aduaneras y judiciales de control del comercio.

Poniendo al lector en contexto, cuando una determinada operación de comercio ilícito de especies CITES es llevada a cabo, el objetivo principal, entre otros, es la retirada de los ejemplares vivos que se encuentren en posesión del infractor de forma que se evite que estos vuelvan a los circuitos comerciales ilegales.

Muchos países del mundo, y también de la propia Unión Europea, no pueden completar sus operaciones contra el comercio ilegal debido a la falta de instalaciones que puedan funcionar como centros de rescate de especímenes decomisados, lo que convierte en estériles las investigaciones llevadas a cabo, y genera una sensación de impunidad en los infractores, al ser procesados y sancionados, pero poder continuar con los ejemplares en su posesión.

Esto adquiere además especial importancia cuando estos especímenes, que quedan a disposición de la Administración, deben ser mantenidos y cuidados en excelentes condiciones de bienestar animal, manejo y control de ejemplares peligrosos.

De todo esto surge entonces la iniciativa española de designar a estos centros de rescate, repartidos por provincias y demarcaciones, especializados por especies, y contribuir con los gastos de mantenimiento con una pequeña contribución económica.

Esto es especialmente importante, puesto que la aportación económica con la que ha contado la autoridad administrativa CITES española, de apenas $317.000 €$ anuales, y su reparto equitativo entre los veinticuatro centros de rescate con los que finalmente se $D$ 
suscribieron estos convenios, apenas ha cubierto un $20 \%$ de los gastos estimados de los especímenes depositados en los centros de rescate a nivel nacional, pudiendo decir sin miedo a equivocarnos que esta actuación ha contado siempre con un matiz altruista por parte de los centros colaboradores con convenio, lo que sin embargo no impidió que el Parlamento Europeo señalara a España como ejemplo en la Conferencia «Breaking point - solving the european wildlife rescue crisis. The need for increased co-ordination and support», de 2018, en la que Alicia Sánchez Muñoz, subdirectora general y responsable del área CITES, desarrolló el proceso seguido y el éxito del modelo mediante los convenios suscritos con los centros de rescate en España.

\subsection{Cooperación con medios informáticos}

Si bien la aplicación del convenio en los diferentes países puede sonar «sencilla» una vez se explica, su materialización no lo es en absoluto, y exige de medios humanos y materiales que exceden de lo imaginado.

Prueba de ello, y como máximo exponente de una buena gestión del convenio, se encuentra la aplicación informática que la autoridad administrativa CITES España ha manejado para la emisión de documentos, registro, comprobación y generación de informes sobre las operaciones comerciales, y los permisos y certificados generados al efecto.

Esta historia comienza en 1998 con la primera aplicación informática con la que contó la autoridad administrativa española, de corte funcional y estructura simple, que pronto sería reemplazada por la aplicación denominada «Panda», diseñada por el personal CITES del área de servicios centrales, que pasaría, en el año 2010, a una nueva versión reformulada, mejor adaptada y con aquellos errores detectados en la utilización de la anterior corregidos y adaptados a un nuevo sistema descentralizado de emisión de los documentos.

Esta nueva aplicación es la conocida como «LincEx», actualmente en su versión número 29 , que combina una exhaustiva y concreta ayuda a la toma de decisiones del tramitador, con la posibilidad de cargar el resultado de las inspecciones, unido al control de la cría en cautividad y de la reproducción artificial nacional en sus diferentes módulos, permitiendo que los operadores puedan solicitar los documentos necesarios de forma telemática.

Esto no ha pasado tampoco desapercibido para otras autoridades, lo que provocó que, en el año 2012, la autoridad administrativa española cediera la aplicación a sus homólogos portugueses e hiciera lo propio en 2014 con la autoridad serbia, expandiendo una vez más el modelo español de gestión del convenio.

\subsection{La contribución económica de España}

Otro elemento, que sin duda no ha trascendido lo suficiente pero que no por ello carece de extraordinario valor, es la colaboración que la autoridad administrativa española ha realizado a lo largo de estos años en el plano económico para ayudar a la correcta implementación del convenio.

Aquí no solo encontramos la aportación económica que España realiza anualmente al Fondo Fiduciario del Convenio CITES, a la que está obligada y se conforma del total atribuido en función del PIB nacional, sino también aquella realizada de forma altruista por $\triangleright$ 
TRAFFIC International, también de forma anual, dirigida a la gestión y mantenimiento de las bases de datos generadas mediante el programa EU TWIX (The European Union Trade in Wildlife Information eXchange).

Otro de los proyectos internacionales más significativos financiados por España fue la traducción al español del libro The evolution of CITES: a reference to the Convention on International Trade in Endangered Species of Wild Fauna and Flora, escrito por el holandés Willem Wijnstekers. Este libro se tradujo por primera vez al español en 1994 bajo el título La CITES y su evolución. Posteriormente, se reeditó en los años siguientes con un nuevo título: La evolución de la CITES. Este proyecto de traducción fue especialmente celebrado por todos los países hispanohablantes.

También se recibió el agradecimiento de todos los organismos y las entidades (museos, asociaciones, centros zoológicos y botánicos, etcétera) a las que se les distribuyeron varios ejemplares del libro con ánimo de difundir y fomentar el conocimiento sobre CITES.

Sería injusto no mencionar aquellas aportaciones que también se han realizado en el periodo previo a cada Conferencia de las Partes, concretamente dirigidas a la UICN (Unión Internacional para la Conservación de la Naturaleza) para colaborar en la traducción de los documentos de las sesiones al español.

\section{Actividades de carácter divulgativo}

Es necesario resaltar, en este punto dedicado al trabajo realizado por la autoridad administrativa CITES en España, aquellas actividades de carácter divulgativo que han perpetrado el lado más visual de la labor que se realiza.
Estas son, entre otras, la publicación de artículos sobre CITES, la organización de exposiciones y el reparto de folletos informativos, que han supuesto, sin lugar a dudas, uno de los instrumentos más efectivos de concienciación social.

\subsection{Publicación de artículos}

A continuación, se recopilan algunos de los artículos más destacados que han sido publicados en el Boletín de Información Comercial Española de la Secretaría de Estado de Comercio del actual Ministerio de Industria Comercio y Turismo:

- Rubio de Urquía, J. (1991). El caso del atún rojo: un precedente conflictivo. Boletín de Información Comercial Española, (2303).

- Pálla Sagüés, O. y Rubio de Urquía, J. (1992). La conferencia de Naciones Unidas sobre medio ambiente y el desarroIlo: aspectos comerciales. Boletín de Información Comercial Española, (2327).

- Valiente Calvo, P. (1993). Convenio CITES. Aplicación en la CEE y en España. Boletín de Información Comercial Española, (2369).

- Núñez Román, M. (2009). 75 Aniversario del SOIVRE. Convenio CITES: su relevancia en el contexto de comercio y medio ambiente. Boletín de Información Comercial Española, (2975).

- Núñez Román, M., Alzás de la Fuente, M. y Sánchez Muñoz, A. (2017). Convenio CITES: Instrumento de Política Comercial Regulador del Comercio de Vida Silvestre. Boletín de Información Comercial Española, (3091). 


\subsection{Exposiciones}

En lo que se refiere a la organización de exposiciones, el trabajo ha consistido en la instalación de vitrinas y cartelería explicativa tanto en las dependencias del propio Ministerio de Industria, Comercio y Turismo como en puertos y aeropuertos (Puerto de Bilbao, 2016; Aeropuerto de Madrid-Barajas, 2010).

Dichas vitrinas contaban, cómo no, con material cedido por la autoridad administrativa, todo ello dispuesto en secciones diferenciadas y con referencia al tipo de comercio realizado sobre esos ejemplares, de modo que pudieran diferenciarse los ejemplares CITES prohibidos en comercio de aquellos que pueden ser comercializados como excepciones. Todo ello de una forma muy didáctica y adaptada al público general.

Asimismo, para hacer llegar estas exposiciones a un público más amplio y variado, su instalación no solo se reservó a las dependencias ya indicadas, sino que también se trasladaron a varios de los centros de rescate que colaboran con la autoridad administrativa CITES para que así pudieran disponer de material de este tipo en apoyo a sus labores de difusión y concienciación con el tráfico de especies protegidas. Algunos de los centros donde se destinaron fueron Loro Parque Fundación, en la isla de Tenerife y la Fundación Natura Parc, situada en las Baleares.

\subsection{Material divulgativo}

Dentro del material divulgativo generado por la autoridad administrativa CITES española, no pueden faltar los trípticos informativos preparados por el personal de servicios centrales, dirigidos a proporcionar información clara, didáctica y práctica sobre CITES, su implementación y elementos fundamentales para el público en general, garantizando el conocimiento y la comprensión del convenio a todas las partes interesadas, de los que se han impreso y repartido más de 3.000 ejemplares distribuidos en todas las delegaciones provinciales y territoriales de comercio, así como traducido al inglés y dispuesto en versión descargable en la página web de la autoridad administrativa principal.

Continuando con esta voluntad de difusión, conocimiento y divulgación del convenio y sus elementos más complejos, la autoridad administrativa CITES preparó la página web www.cites.es, dedicada a dar información actualizada sobre legislación, autoridades, permisos y certificados, estadísticas, noticias y cuestiones de interés, siempre con un matiz práctico y explicativo, en varios idiomas, y con accesos directos a las solicitudes telemáticas o a los contactos de la red territorial, convirtiéndose en la página de referencia de los sectores interesados en la materia a nivel nacional.

Además, los funcionarios de la autoridad administrativa, ya sea como personal al servicio de esta o a título personal —en este último caso, siempre que las circunstancias lo han permitido-, han atendido gratamente a las consultas y peticiones de particulares con el fin de ampliar la comprensión del funcionamiento y los objetivos del convenio a toda clase de interesados, desde estudiantes universitarios y doctorandos hasta autores de artículos especializados y prensa general.

Todas estas acciones formativas, asistenciales, cooperativas y divulgativas han contribuido al fortalecimiento de la aplicación del CITES y parte de su éxito se debe al acierto en el diseño y orientación de estas. 


\section{Colaboración internacional}

En relación a la colaboración internacional, tanto la autoridad administrativa como la científica han colaborado muy activamente en misiones y asesorías a nivel internacional. A continuación se hará un repaso por años de las más importantes.

\subsection{Misiones}

Año 1994, misión a Tailandia para inspeccionar viveros tailandeses tras su cierre por comercio ilegal, organizada por la Secretaría CITES, realizada con los representantes de: Reino Unido (representante de Europa), Oceanía (presidente del Comité de Flora), Alemania, Países Bajos y TRAFFIC.

Año 1994, misión a México para visitar viveros mexicanos y diversos jardines botánicos (UNAM, Cuernavaca, Xalapa), organizada por las autoridades CITES de México, realizada con los representantes de Reino Unido (representante de Europa), Alemania, Suiza, TRAFFIC y UICN.

Año 1994, misión a Estados Unidos para visitar el Fairchild Botanic Garden de Miami y el Centro de Inspección Fitosanitaria y CITES de Plantas situado en Miami, organizada por las autoridades fitosanitarias de EE. UU.

Año 1999, misión a Cuba. Fue la primera de estas misiones en las que participó la asesoría de fauna de la autoridad científica española. La Comisión Europea había sido invitada por Cuba para mostrarle la gestión que hacía de la tortuga carey (Eretmochelys imbricata) en sus aguas, ya que estaba intentando que se le permitiera la comercialización de unos 5.000 kg de carey almacenado proveniente de la captura anual que el Gobierno cubano realizaba de esta especie. El director de la asistencia técnica para fauna viajó con otros colegas de la UE, incluyendo una representante de la autoridad administrativa española, para conocer la gestión y medidas de control diseñados por Cuba a los efectos mencionados.

Año 2003, misión a Paraguay con miembros de la Secretaría CITES, incluyendo al entonces secretario general de CITES, Willem Wijnstekers. Se trataba de conocer in situ el comercio de especies CITES de Paraguay, especialmente de reptiles, a raíz de repetidas denuncias de comercio ilegal que había recibido la Secretaría CITES. Dado el importante comercio de pieles de reptiles entre España y Paraguay, España aportó parte de la financiación necesaria para realizar esta misión, lo que abrió la puerta a la participación de un miembro de las autoridades CITES españolas. Dado que se trataba principalmente de asuntos de legalidad y control, la Secretaría CITES aportó sus más veteranos expertos en este ámbito, por lo que se decidió que la participación española fuera en los aspectos científicos. A raíz de esta visita, Paraguay reconoció irregularidades en su comercio de especies CITES y estableció una moratoria para ese comercio.

Año 2004, misión a Paraguay para impartir un taller de capacitación a sus autoridades CITES. Organizado por la Secretaría CITES y las autoridades CITES españolas, en colaboración con las autoridades de Paraguay, contó con la participación por parte de España de sus autoridades administrativa y científica, siendo el papel de esta última la formación en aspectos científicos en CITES y, en particular, en la elaboración de dictámenes de extracción no perjudicial.

En un caso similar al anterior, misión a Bolivia para impartir un taller de capacitación a sus autoridades CITES. Organizado por la Secretaría CITES y las autoridades CITES de $\triangleright$ 
Bolivia, contó con la participación por parte de España de sus autoridades administrativa y científica, siendo el papel de esta última la formación en aspectos científicos en CITES y, en particular, en la elaboración de dictámenes de extracción no perjudicial.

Año 2005, en la tónica de las anteriores, misión a Perú para participar en un taller de capacitación con sus autoridades CITES. Organizado por la Secretaría CITES y las autoridades CITES de Perú, contó con la participación por parte de España de sus autoridades administrativa y científica, siendo el papel de esta última la formación en aspectos científicos en CITES y, en particular, en la elaboración de dictámenes de extracción no perjudicial.

Año 2006, misión a Perú. Esta fue una misión de la Unión Europea para conocer la situación de la caoba de hoja ancha. Durante la misma, se mantuvieron reuniones con las autoridades CITES, autoridades de control, organizaciones no gubernamentales y otros actores implicados en el manejo de la especie.

Año 2007, misión a Bolivia para participar en un taller de capacitación con sus autoridades CITES. Organizado por la Secretaría CITES y las autoridades CITES de Bolivia, contó con la participación por parte de España de su autoridad científica, ocupándose de aspectos científicos en CITES y, en particular, de la elaboración de dictámenes de extracción no perjudicial.

Año 2008, misión a Indonesia. Con una temática diferente a la capacitación, España participó en la misión de la Unión Europea a Indonesia en el año 2008 a través de su autoridad científica. Dado el gran comercio importador de la UE desde Indonesia, en especial de corales y reptiles, la Comisión Europea organizó esta misión junto con las autoridades CITES de Indonesia para conocer sobre el terreno aspectos científicos y de control del comercio de especies CITES. La misión se centró hacia el conocimiento de la cría en cautividad de reptiles y la maricultura de corales. Participaron la Comisión Europea, Reino Unido, Francia y España.

Año 2010, misión a Líbano para participar en un taller sobre bienestar animal, CITES y zoos, organizado por la Comisión Europea y el Ministerio de Agricultura de Líbano. España estuvo presente en esta misión a través de su autoridad científica.

Año 2010, misión a Perú para participar en un taller organizado por sus autoridades CITES sobre capacitación para la elaboración de dictámenes de extracción no perjudicial. La presencia de España fue a través de su autoridad científica.

Año 2017, misión a Colombia financiada por la Unión Europea como consecuencia de diversas informaciones recibidas sobre posibles irregularidades en la producción de pieles de cocodrílidos en las granjas de dicho país. En esta misión participaron España y Francia.

\subsection{Twinning de Turquía}

Entre 2011 y 2013 España desarrolló en Turquía un proyecto de hermanamiento de la Unión Europea (twinning) denominado «Fortalecimiento de las capacidades institucionales para la aplicación del CITES en Turquía» (Strengthening the institutional capacity on CITES implementations). El proyecto se desarrolló bajo ocho componentes que comprendían veinticinco actividades y se contó con la participación de veintisiete expertos.

Entre las actividades cabe destacar la elaboración de guías de procedimiento, como la guía para la disposición de especímenes confiscados, la realización de seis cursos en los $\triangleright$ 
que se formaron 269 personas, la traducción al turco de diez guías de identificación y 524 fichas identificativas, así como la elaboración de nuevas fichas de identificación para especies relevantes en Turquía y la elaboración de material divulgativo.

\subsection{El Máster CITES}

Pero, sin duda, una de las grandes contribuciones de España al Convenio CITES ha sido la organización de un máster, único en el mundo, que constituiría la joya de la corona en la formación impartida en esta materia a autoridades de todo el mundo, el Máster en Gestión y Conservación de Especies en Comercio: el Marco Internacional.

La idea del máster nació en Roma en diciembre de 1996. En la sesión 37. ${ }^{\text {a }}$ del Comité Permanente (CP) de CITES participaron el delegado de España, Javier Rubio de Urquía, representando a la autoridad administrativa, y la presidenta del Comité de Flora, Margarita África Clemente Muñoz. Ambos señalaron la urgencia de fortalecer las capacidades y aplicación del CITES de los países de origen de fauna y flora silvestre. Una necesidad que habían detectado hacía tiempo con creciente preocupación. Al terminar la reunión surgió la idea de poner en marcha una iniciativa de excelencia para las Partes que estaban demandando una formación intensa y extensa. Pocos meses después, la Dra. Clemente presentó, como directora, una propuesta de máster a la Universidad Internacional de Andalucía (UNIA), respaldada por la autoridad administrativa española, que fue aceptada y recibió los apoyos de la Secretaría CITES y del CP. Así fue posible anunciar la primera edición del máster para el curso 1998; la última edición se realizó en 2018.
El Máster CITES ha sido impartido en trece ocasiones y estuvo ligado a un programa para la obtención del Diploma de Estudios Avanzados y la Suficiencia Investigadora en colaboración con la Universidad de Córdoba.

Por sus características, es único en el mundo y goza de un amplio reconocimiento internacional gracias al esfuerzo de muchas personas que han impartido docencia en él y al apoyo incondicional y permanente en el tiempo de la autoridad administrativa española. España ha recibido un amplísimo reconocimiento de la Secretaría CITES y de la Conferencia de las Partes por llevar a cabo esta formación de excelencia.

El objetivo general es proporcionar una formación de alta calidad, especializada en los fundamentos científicos, las técnicas e instrumentos que permiten la aplicación y desarrollo de CITES. Las aulas, dependencias y personal de la sede de Antonio Machado en Baeza han propiciado un ambiente de trabajo que destaca por su multidisciplinariedad y multiculturalidad.

Tras trece ediciones, este máster ha proporcionado formación especializada de calidad a 350 personas de 97 países procedentes de África, América, Asia, Europa y Oceanía. Los resultados de esta iniciativa han sido publicados en 2019 en el libro de UNIA titulado Máster CITES: un caso de éxito en la cooperación española ( $A$ success story of Spanish cooperation), volumen coordinado por Margarita África Clemente Muñoz y Javier Rubio de Urquía y publicado por la Agencia Española de Cooperación Internacional para el Desarrollo (AECID) ${ }^{1}$.

Sin ahondar especialmente en cuestiones que se han abordado en apartados anteriores, no es posible continuar sin hacer mención a aquella formación que ha traspasado nuestras $\triangleright$

1 https://cites.org/sites/default/files/esp/com/sc/70/inf/S-SC70-Inf-10.pdf 
fronteras, y no precisamente por traer a personal CITES de terceros países a España, como en el caso del máster, sino por movilizarla y desplazarla para brindar sus conocimientos a Turquía, mediante el programa Twinning del año 2009, organizado e impartido por la autoridad administrativa española, o el programa Twinning de Serbia del año 2013, en el que España participaba a través de D. Antonio Galilea Jiménez, personal SOIVRE del área CITES de los servicios centrales y la máxima referencia nacional en materia de aplicación y observancia del Convenio CITES en la autoridad administrativa CITES España.

\section{Perspectivas de futuro}

La convención es un instrumento comercial clave al servicio de la conservación y el uso sostenible de las especies. No debería confundirse con la preservación o no uso, y velar para que los países de origen, en gran medida en vías de desarrollo o pobreza extrema, puedan utilizar sus recursos de forma apropiada y conservarlos para las generaciones futuras. No es posible negar el carácter comercial del Convenio CITES, ya que precisamente su fundamento, así como los instrumentos y mecanismos que utiliza para su aplicación, son básicamente comerciales. El éxito del mismo es la regulación comercial.

El papel de España en CITES puede calificarse de brillante durante todos estos años, en especial por la dedicación, formación, profesionalidad y trabajo de las personas que han estado desempeñando la autoridad administrativa española hasta 2021. España ha obtenido un reconocimiento relevante en CITES y especialmente de las partes de países en desarrollo a los que se ha proporcionado formación de alta calidad y gran apoyo en la aplicación de la convención.
Lamentablemente, la presión actual de organizaciones no gubernamentales de carácter internacional, de ideologías puramente conservacionistas y animalistas, está cambiando la visión inicial de CITES y los objetivos para los que el convenio fue creado. El concepto de preservación a ultranza y bienestar animal está ganando terreno al de uso sostenible, y esto se está reflejando claramente en las propuestas presentadas y en los resultados de las últimas Conferencias de las Partes.

Las tesis más conservacionistas son las que están prevaleciendo, castigando a aquellos países que, a pesar de realizar grandes esfuerzos en combatir el comercio ilegal y aplicar políticas muy eficientes en la conservación de sus recursos, ven que una y otra vez sus propuestas para poder beneficiarse de su buena gestión van siendo rechazadas. A pesar de que los datos demuestran claramente que el comercio ilegal representa un ínfimo porcentaje frente al legal, se ha generalizado la creencia de que todo comercio implica un tráfico ilegal.

Una de las cuestiones que resultan más preocupantes es el hecho de que esta presión esté desencadenando cambios en la gestión de CITES en bastantes países, siendo las tesis puramente conservacionistas y la asociación de «comercio» con «ilegal» las que están influyendo en dichos cambios. Cuando se producen modificaciones en unos sistemas de gestión tan complejos, como lo es la gestión de CITES, ya sea por motivación política o ideológica, no está claro el impacto o las repercusiones que pueden tener en el funcionamiento de estos. Lo deseable en estos casos sería que siempre que se realizasen cambios de gran calado fueran asociados a una previa evaluación de dichos sistemas para justificar si estos son necesarios e imponer acciones correctivas solo si así fuese preciso. 
En nuestro país podemos estar orgullosos de haber tenido un sistema de gestión que ha funcionado durante 35 años de manera exitosa y eficiente. Un sistema que ha costado mucho tiempo y enormes esfuerzos y recursos económicos del Estado.

Por el bien de las especies involucradas y el desarrollo sostenible de los países que todavía cuentan con valiosos recursos, confiamos en que el Convenio CITES siga evolucionando sin desviarse de los objetivos para los que fue ideado.

\section{Conclusión}

En la aplicación de CITES, la memoria histórica es fundamental. En el caso de España, la existencia de un conjunto de expertos que puedan distinguirse del resto por contar con tal amplia experiencia en la gestión del convenio fue posible gracias a la estabilidad que se mantuvo por más de treinta años en la asignación de competencias administrativas.

Tal y como se ha señalado en la introducción, CITES es un convenio complejo, y este es uno de los motivos por el que son muchas las oportunidades que se presentan de emprender actuaciones de colaboración en múltiples ámbitos. No obstante, es necesario contar con expertos capaces de apreciar qué actuaciones son las que tendrán un mayor impacto positivo.

Así pues, es crucial contar con un bagaje a la par que con un conocimiento de la situación actual para poder identificar cuáles son las necesidades reales a la hora de mejorar la aplicación.

Desde el comienzo de la aplicación del convenio en España las competencias en materia de representación y gestión han sido desempeñadas por los mismos dos cuerpos de funcionarios. Estos son el Cuerpo de Inspectores del SOIVRE y el Cuerpo de Ingenieros Técnicos del SOIVRE, los cuales han mantenido hasta la fecha el ejercicio de las competencias de autoridad administrativa principal en España. Por ende, la historia de la gestión de este convenio en España, desde su inicio hasta hoy, la han protagonizado estos funcionarios. Gracias a su versatilidad dentro de la esfera del comercio fue posible que pudieran asumir la gestión del CITES cuando este aún no era tan conocido como es ahora.

Es curioso cómo España, sin ser uno de los países pioneros en firmar el convenio, se convirtió en un ejemplo modélico con dos cuerpos de funcionarios altamente competentes en la gestión del CITES, siendo las claves de esta profesionalización la estabilidad de la que ha gozado la autoridad administrativa principal, que ha descansado siempre bajo la Secretaría de Estado de Comercio, así como haber contado con un conjunto de funcionarios multidisciplinares, tal y como se caracterizan los inspectores e ingenieros técnicos del SOIVRE.

Cabe decir que el reconocimiento que ha recibido esta autoridad por parte del resto de expertos internacionales del CITES ya es motivo suficiente para poner en valor todo el trabajo realizado y darle a esta autoridad el crédito que se merece por su buen criterio y actuación durante todos estos años. La intensa dedicación y absoluta fidelidad al objetivo de consecución de un equilibrio entre conservación y uso sostenible de las especies hará que las futuras generaciones puedan sentirse orgullosas del cometido logrado.

\section{Bibliografía}

Universidad Internacional de Andalucía. (2019). Máster CITES: un caso de éxito en la cooperación española. Agencia Española de Cooperación Internacional para el Desarrollo. https:// bibliotecadigital.aecid.es/bibliodig/i18n/ catalogo_imagenes/grupo.do?path=1026184 
